\title{
The surgical outcome of decompression alone versus decompression with limited fusion for degenerative lumbar scoliosis
}

\author{
Kenji Masuda, MD, Takayuki Higashi, MD, Katsutaka Yamada, MD, PhD, Tatsuhiro Sekiya, MD, and \\ Tomoyuki Saito, MD, PhD
}

Department of Orthopaedic Surgery, Yokohama City University School of Medicine, Yokohama, Japan

\begin{abstract}
OBJECTIVE The aim of this study was to assess the usefulness of radiological parameters for surgical decision-making in patients with degenerative lumbar scoliosis (DLS) by comparing the clinical and radiological results after decompression or decompression and fusion surgery.

METHODS The authors prospectively planned surgical treatment for 298 patients with degenerative lumbar disease between September 2005 and March 2013. The surgical method used at their institution to address intervertebral instability is precisely defined based on radiological parameters. Among 64 patients with a Cobb angle ranging from $10^{\circ}$ to $25^{\circ}$, 57 patients who underwent follow-up for more than 2 years postoperatively were evaluated. These patients were divided into 2 groups: those in the decompression group underwent decompression alone $(n=25)$, and those in the fusion group underwent decompression and short segmental fusion $(n=32)$. Surgical outcomes were reviewed, including preoperative and postoperative Cobb angles, lumbar lordosis based on radiological parameters, and Japanese Orthopaedic Association (JOA) scores.
\end{abstract}

RESULTS The JOA scores of the decompression group and fusion group improved from $5.9 \pm 1.6$ to $10.0 \pm 2.8$ and from $7.2 \pm 2.0$ to $11.3 \pm 2.8$, respectively, which was not significantly different between the groups. At the final followup, the postoperative Cobb angle in the decompression group changed from $14^{\circ} \pm 2.9^{\circ}$ to $14.3^{\circ} \pm 6.4^{\circ}$ and remained stable, while the Cobb angle in the fusion group decreased from $14.8^{\circ} \pm 4.0^{\circ}$ to $10.0^{\circ} \pm 8.5^{\circ}$ after surgery.

CONCLUSIONS The patients in both groups demonstrated improved JOA scores and preserved Cobb angles after surgery. The improvement in JOA scores and preservation of Cobb angles in both groups show that the evaluation of spinal instability using radiological parameters is appropriate for surgical decision-making.

https://thejns.org/doi/abs/10.3171/2018.1.SPINE17879

KEYWORDS degenerative lumbar scoliosis; decompression; short segmental fusion; criteria for intervertebral instability

$\mathrm{S}$ EVERAL surgical options are available for patients with degenerative lumbar scoliosis (DLS). ${ }^{2,12,15,16}$ The recommended surgery for DLS with a coronal curve of less than $30^{\circ}$ is posterior decompression and short fusion without correction of the curve. ${ }^{15}$ For a coronal curve of more than $30^{\circ}$ that requires correction of global coronal balance, a long corrective fusion should be performed. ${ }^{16}$ The classification of adult spinal deformity (Schwab adult deformity classification) is well established, easy to use, and consistent with the treatment of adult scoliosis. ${ }^{12}$ Some surgeons recommend performing fusion with instrumentation to prevent curve collapse. ${ }^{4,18}$ However, the surgical treatment of DLS with stenosis in elderly patients is often challenging because these patients frequently have medical comorbidities, which are associated with higher complication rates. ${ }^{12}$ DLS includes a diversity of symptoms, such as back pain, claudication, and neurological deficits. The management of DLS is characterized by significant variability because of the absence of a clear and evidencebased approach to treatment. The appropriate indications for the optimal treatment of DLS have yet to be defined. We hypothesized that the outcome of decompression surgery for mild DLS, based on our definition by radiological measurements of instability, would be as good as decompression with limited fusion. We prospectively analyzed the clinical outcomes of surgical treatment for patients

ABBREVIATIONS DLS = degenerative lumbar scoliosis; JOA = Japanese Orthopaedic Association; TLIF = transforaminal lumbar interbody fusion

SUBMITTED August 16, 2017. ACCEPTED January 24, 2018.

INCLUDE WHEN CITING Published online June 1, 2018; DOI: 10.3171/2018.1.SPINE17879. 
Surgical treatment for degenerative lumbar disease in our hospital $(n=298)$

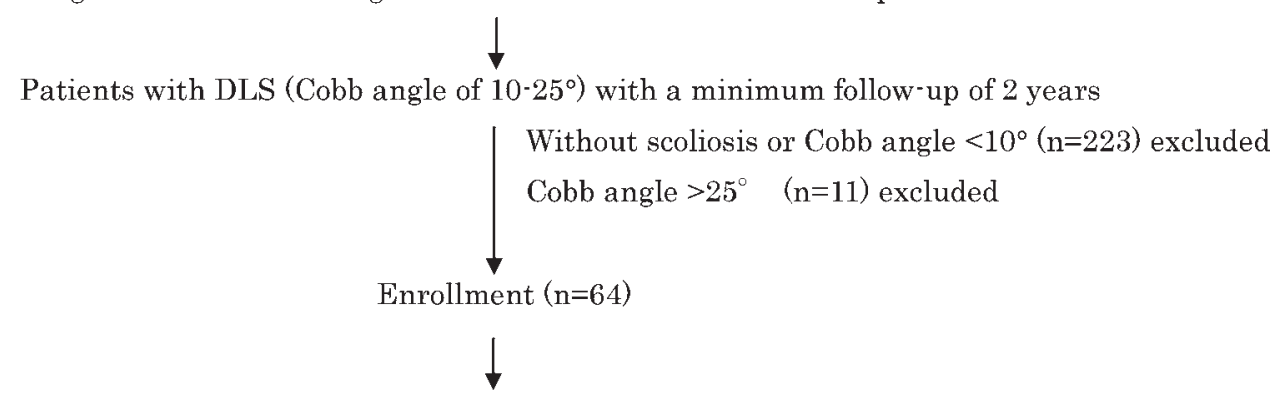

Treatment group

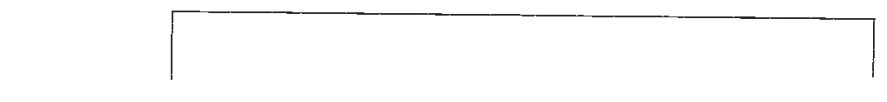

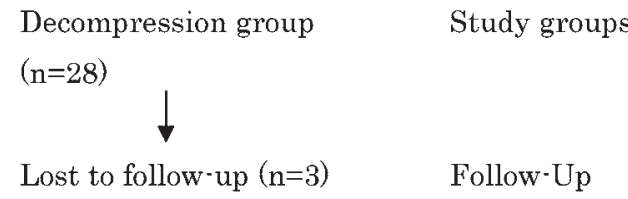

Never returned to the hospital

$$
\text { Analyzed ( } \mathrm{n}=25)
$$

Analysis

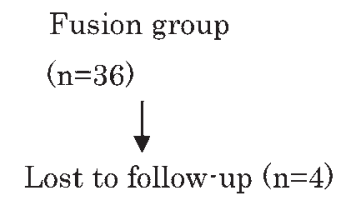

Never returned to the hospital

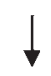

Analyzed $(n=32)$

FIG. 1. Patient sampling and selection. Flowchart illustrating the enrollment of patients into the study. Patients with degenerative lumbar scoliosis (Cobb angle $10^{\circ}-25^{\circ}$ ) with a minimum follow-up of 2 years are included and divided into 2 surgical groups. The patients in the decompression group $(n=25)$ and the patients in the fusion group $(n=32)$ are then analyzed.

with mild DLS. All patients underwent decompression surgery with or without limited segmental fusion, depending on radiological parameters for segmental spinal instability determined by preoperative coronal and lateral radiographs.

The purpose of this study was to compare the clinical and radiological results of decompression alone versus decompression with short fusion for mild DLS and to assess the usefulness of radiological parameters for surgical decision-making in these patients.

\section{Methods}

This study was approved by the ethics research board of the Yokohama City University School of Medicine. All patients provided informed consent for their participation in the study.

From a total of 298 patients in whom we prospectively planned surgical treatment for degenerative lumbar disease between September 2005 and March 2013 in our hospital, we chose patients with lumbar canal stenosis and mild DLS for this study. We excluded 223 patients without scoliosis or whose Cobb angle was less than $10^{\circ}$, as well as 11 patients whose Cobb angle was more than $25^{\circ}$. Sixty-four patients whose Cobb angle ranged from $10^{\circ}$ to $25^{\circ}$ were enrolled in this study (Fig. 1), and we were able to monitor 57 patients (89\%) for a minimum of 2 years postoperatively. The surgical modality was determined based on the degree of spinal instability in the radiological findings.
Radiological evaluation was performed based on functional lateral radiographs in a lateral decubitus position and anteroposterior lumbar radiographs in a standing position. A lateral view was obtained in the lateral decubitus position because maximal-effort flexion-extension radiographs were obtained in the patient without pelvic restraint. ${ }^{1}$

The radiological parameters were measured manually on preoperative radiographs using digital calipers (Fig. 2). The dynamic parameters included segmental lordosis during lumbar extension, anterior translation during flexion, posterior translation during extension, and disc wedging. Our definition of radiological intervertebral instability was based on the report by Aota et al., which established 5 criteria for intervertebral instability: 1) $15^{\circ}$ or greater segmental range of motion, 2) $3 \mathrm{~mm}$ or greater anterior translation, 3) $3 \mathrm{~mm}$ or greater posterior translation in the sagittal plane, 4) $5^{\circ}$ or greater disc wedging, and 5) $3 \mathrm{~mm}$ or greater lateral translation in the coronal plane (Fig. 2). ${ }^{1}$

All patients underwent neurological decompression. Patients who were 75 years or older whose spinal column met 3 or more of the 5 parameters and patients younger than 75 years whose spinal column met 2 or more of the parameters also underwent limited segmental fusion. Using these criteria, 25 patients underwent decompression alone and 32 patients underwent decompression and fusion.

\section{Operative Procedures}

All patients underwent decompression surgery at the 
A

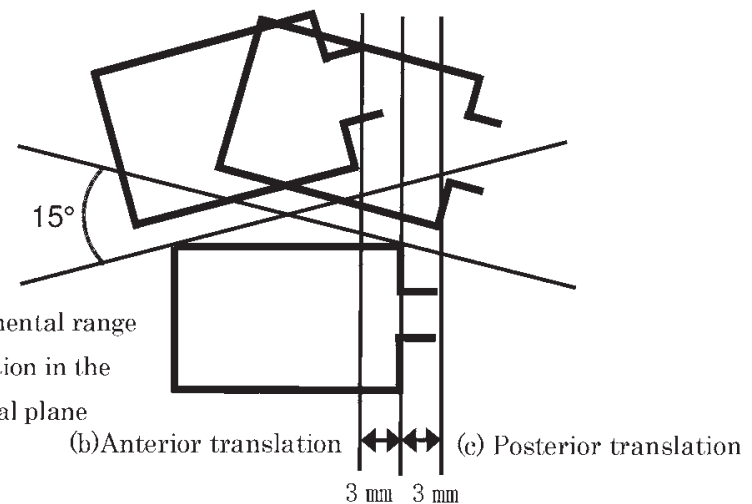

(a) Segmental range

of motion in the sagittal plane

$3 \mathrm{mmll} 3 \mathrm{~mm}$

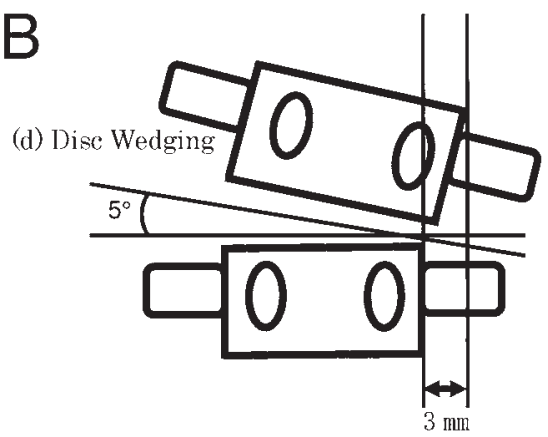

(c) Lateral translation

FIG. 2. Illustrations representative of radiographs used for evaluating spinal instability. A: Functional lateral radiographs in flexion and extension are obtained in the lateral decubitus position; measurements of segmental range of motion in the sagittal plane (a), anterior translation with flexion (b), and posterior translation with extension (c). B: Anteroposterior radiographs in a standing position; measurements of disc wedging (d) and lateral translation (e).

identified level of spinal stenosis. In the decompression group, 19 patients underwent fenestration by a lumbar spinous process-splitting technique, and 6 patients underwent conventional fenestration.

Seventeen patients underwent a 1-level decompression, 6 patients had a 2-level, and 2 patients had a 3-level decompression. In a lumbar spinous process-splitting technique, the lamina was exposed by longitudinally splitting the spinous process into halves, while muscular and ligamentous attachments to the spinous process remained intact. This procedure allows better visualization and a wider working space while minimizing damage to the posterior lumbar supporting structures. ${ }^{19}$ In the fusion group, all patients underwent thorough decompression, followed by transforaminal lumbar interbody fusion (TLIF) of the affected level(s). Sixteen patients underwent a 1-level fusion, 15 patients a 2-level, and 1 patient a 3-level fusion. The pedicle screws were compressed along a lordotic rod to restore lumbar lordosis, although the realignment of spinal imbalance was not attempted while performing instrumentation.

We obtained Japanese Orthopaedic Association (JOA) scores for all patients both preoperatively and at the final follow-up. The JOA score is a clinical evaluation scoring system for low-back pain syndrome, including subjective
TABLE 1. Portion of the scoring system of the JOA for low-back pain (JOA score)

\begin{tabular}{|c|c|}
\hline Symptoms \& Signs & Score \\
\hline \multicolumn{2}{|l|}{ Subjective symptoms } \\
\hline \multicolumn{2}{|l|}{ Low-back pain } \\
\hline None & 3 \\
\hline Occasional, mild & 2 \\
\hline Occasional, severe & 1 \\
\hline Continuous, severe & 0 \\
\hline \multicolumn{2}{|l|}{ Leg pain \&/or tingling } \\
\hline None & 3 \\
\hline Occasional, slight & 2 \\
\hline Occasional, severe & 1 \\
\hline Continuous, severe & 0 \\
\hline \multicolumn{2}{|l|}{ Gait } \\
\hline Normal & 3 \\
\hline Able to walk $>500 \mathrm{~m}$ but results in symptoms & 2 \\
\hline Unable to walk $>500 \mathrm{~m}$ & 1 \\
\hline Unable to walk >100 m & 0 \\
\hline \multicolumn{2}{|l|}{ Clinical signs } \\
\hline \multicolumn{2}{|l|}{ Straight leg-raise test } \\
\hline Normal & 2 \\
\hline $30^{\circ}-70^{\circ}$ & 1 \\
\hline$<30^{\circ}$ & 0 \\
\hline \multicolumn{2}{|l|}{ Sensory disturbance } \\
\hline None & 2 \\
\hline Slight disturbance (not subjective) & 1 \\
\hline Marked disturbance & 0 \\
\hline \multicolumn{2}{|l|}{ Motor disturbance } \\
\hline Normal & 2 \\
\hline Slight weakness (MMT 4) & 1 \\
\hline Marked weakness (MMT 0-3) & 0 \\
\hline \multicolumn{2}{|l|}{ Urinary bladder function } \\
\hline Normal & 0 \\
\hline Mild dysuria & -3 \\
\hline Severe dysuria & -6 \\
\hline
\end{tabular}

MMT = manual muscle testing.

In the JOA evaluation system for low-back pain syndrome (JOA score), a score of 15 is classified as normal.

symptoms, clinical signs, restrictions in activities of daily living, and urinary bladder function. We used a modification of the JOA score, omitting the section on the restriction of activities of daily living for this study (Table 1). ${ }^{13}$ A normal score is 15 points. The recovery rate was calculated using the following formula: recovery rate (\%) $=($ postoperative JOA score - preoperative JOA score $) /(15$ - preoperative JOA score) $\times 100$.

We also evaluated patient radiographs. Anteroposterior and lateral radiographs obtained preoperatively, postoperatively, and at final follow-up were reviewed. Both Cobb angle and lumbar lordosis were assessed. Lumbar lordosis was measured as the angle between the inferior endplate 
TABLE 2. Comparison of demographic and clinical characteristics of 57 patients treated with decompression and fusion for DLS

\begin{tabular}{lccc}
\hline & $\begin{array}{c}\text { Decompression } \\
\text { Group }\end{array}$ & $\begin{array}{c}\text { Fusion } \\
\text { Group }\end{array}$ & $\begin{array}{c}\mathrm{p} \\
\text { Value }\end{array}$ \\
\hline No. of patients & 25 & 32 & \\
\hline Sex, $\mathrm{n}(\%)$ & $14(56 \%)$ & $12(38 \%)$ & \\
\hline Male & $11(44 \%)$ & $20(62 \%)$ & \\
\hline Female & $73 \pm 7.1(61-86)$ & $68 \pm 9.0(40-81)$ & $<0.05$ \\
\hline $\begin{array}{l}\text { Mean age at op in yrs } \\
\text { (range) }\end{array}$ & $66 \pm 30$ & $60 \pm 26.7$ & $\mathrm{NS}$ \\
\hline $\begin{array}{l}\text { Mean follow-up period } \\
\text { in mos }\end{array}$ & $14 \pm 2.9^{\circ}$ & $15 \pm 4.0^{\circ}$ & $\mathrm{NS}$ \\
\hline $\begin{array}{l}\text { Mean preop Cobb angle } \\
\text { Mean preop JOA score }\end{array}$ & $5.9 \pm 1.6$ & $7.2 \pm 2.0$ & $<0.05$ \\
\hline $\begin{array}{l}\text { Mean preop lumbar } \\
\text { lordosis }\end{array}$ & $27.5 \pm 13.4$ & $27.8 \pm 15.2$ & $\mathrm{NS}$ \\
\hline
\end{tabular}

NS $=$ not significant.

Mean values are presented as the mean \pm SD .

of T12 and the superior endplate of S1. We also evaluated surgical complications and additional surgeries related to complications.

Statistical analysis was performed using IBM SPSS (version 24, IBM Corp.). We used paired and unpaired ttests. The level of significance was defined as $p<0.05$.

\section{Results}

Radiological intervertebral instability was measured for 85 segments in 57 patients. In the coronal plane, disc wedging was most common and seen in 61 segments $(72 \%)$, followed by lateral translation in 52 segments $(61 \%)$. In the sagittal plane, rotational mobility was seen in 34 segments ( $40 \%)$, followed by anterior translation in 28 (33\%), and posterior translation in $7(8 \%)$.

The mean age of the patients who underwent decompression alone was $73 \pm 7.1$ years (Table 2 ). There were 14 men and 11 women in the decompression group. The mean \pm SD age of the patients who underwent decompression and fusion was $68 \pm 9.0$ years. There were 12 men and 20 women in the fusion group.

The mean preoperative JOA scores for the decompression group and fusion group were $5.9 \pm 1.6$ and $7.2 \pm$ 2.0, respectively (Fig. 3). The JOA scores improved significantly to $10 \pm 2.8$ and $11.3 \pm 2.8$, respectively, at final follow-up. The net change in both groups after surgery did not differ significantly. The recovery rates were $45 \%$ and $53 \%$, respectively, and there was no statistically significant difference between the groups.

The preoperative Cobb angles of the decompression group and fusion group were $14^{\circ} \pm 2.9^{\circ}$ and $14.8^{\circ} \pm 4.0^{\circ}$, respectively (Fig. 4). The postoperative Cobb angles were $13.2^{\circ} \pm 4.7^{\circ}$ and $8.5^{\circ} \pm 6.1^{\circ}$, respectively, and the Cobb angles at the final follow-up were $14.3^{\circ} \pm 6.4^{\circ}$ and $10.0^{\circ}$ $\pm 8.5^{\circ}$, respectively. The postoperative Cobb angle of the decompression group did not progress significantly during the follow-up period. Complications, such as nerve injury,

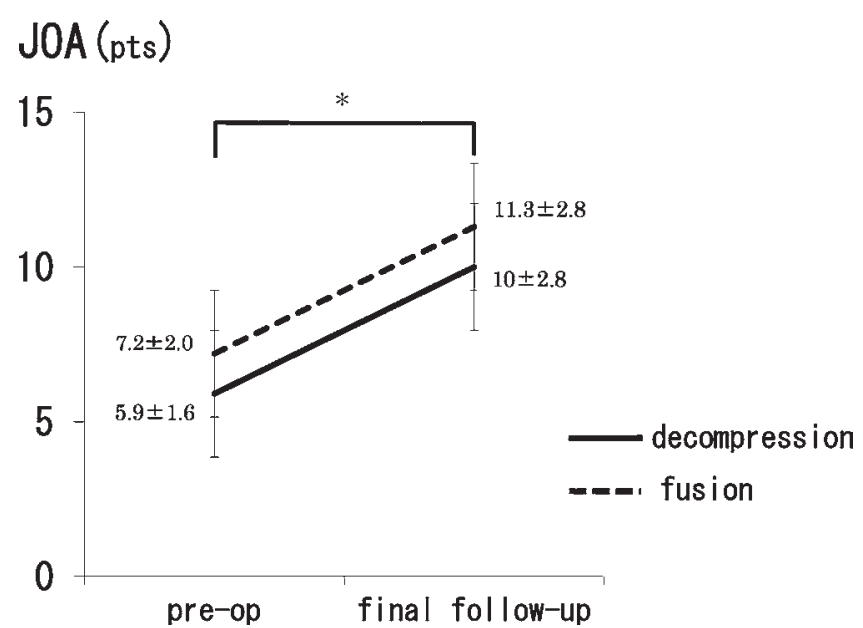

FIG. 3. Mean JOA scores in the decompression and fusion groups (preoperatively and at final follow-up). The mean JOA scores show significant improvement postoperatively in both groups. Data are presented as the mean $\pm S D .{ }^{*} p<0.05$. Pts $=$ points.

infection, or hematoma, were not observed. Two patients in the decompression group underwent a second operation. One patient developed anterior translation and rotatory instability of the decompressed level, the L3-4 level on radiographic evaluation, and complained of lumbar back pain and claudication 6 months after decompression surgery. The patient underwent TLIF of the affected level 1 year after the decompression surgery. Another patient developed intervertebral disc protrusion at the decompressed level without radiological instability. The patient underwent discectomy 1 year after the decompression surgery.

The Cobb angle in the fusion group decreased significantly immediately after surgery and did not progress until final follow-up. The Cobb angle in the decompression group did not change immediately after surgery and did not progress during the follow-up period. Lumbar lordosis in both groups did not change significantly after surgery (Fig. 5).

In the fusion group, 2 patients developed a deep infection in the early postoperative phase and underwent surgical debridement. One patient who underwent TLIF from L3 to L5 developed lower adjacent-segment degeneration and complained of low-back pain and claudication. The patient underwent additional fusion surgery at the L5-S1 level 1 year after the first operation. One patient who underwent TLIF from L3 to L5 developed upper adjacentsegment degeneration and complained of low-back pain. The patient underwent additional fusion surgery at the L2-3 level 1 year after the first operation.

\section{Discussion}

A diagnosis of DLS with lumbar spinal stenosis makes surgical decision-making difficult because DLS can exhibit various neurological symptoms and complex coronal and sagittal findings on radiographs. Surgical options for mild DLS include decompression alone or decompression and limited fusion within the deformity. Surgical re- 


\section{Cobb angle $\left(^{\circ}\right)$}

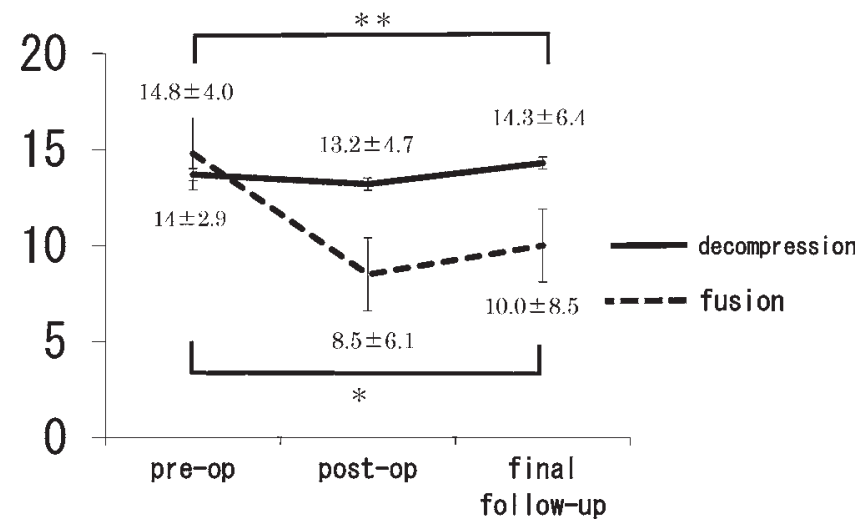

FIG. 4. Mean Cobb angle in the decompression and fusion groups (preoperatively, postoperatively, and at final follow-up). This graph shows that the postoperative Cobb angle in the decompression group does not progress significantly during the follow-up period. The Cobb angle in the fusion group decreases significantly immediately after the surgery and does not progress significantly during the follow-up period. There is no significant postoperative progression of the Cobb angle in either group. Data are presented as the mean \pm SD. ${ }^{*} p<0.05 ;{ }^{* *}$ not significant.

alignment of the deformity is necessary for severe DLS. Several factors, including instability, must be considered when choosing the optimal surgical method. In this study, we focused on coronal curve progression after surgical treatment of mild DLS using our radiological instability criteria.

Simmons suggested that the neural elements as well as the stability and alignment of the vertebral column are important in deciding surgical treatment in patients with spinal stenosis associated with DLS. ${ }^{16}$ Neglect of either of these components will lead to inadequate results and less than ideal clinical outcomes. However, the optimal surgical treatment is unclear. Postacchini and Gupta reported that decompression surgery alone can be performed in patients with minor curves and no back pain. ${ }^{7,14}$ Some authors have suggested that simple decompression is sufficient, provided that there is no significant kyphosis, olisthesis, or back pain because surgery can lead to further collapse, instability, continued back pain, and exacerbation of neural symptoms. ${ }^{16,18}$ Daubs et al. reported that decompression with limited fusion for mild DLS prevents early return of stenotic symptoms compared with decompression alone and recommended limited fusion for mild DLS. ${ }^{5}$ Liu et al. reported that it is preferable to perform fixation and fusion in conjunction with decompressive laminectomy when 2 or more segments are involved, and that, although simple decompression is indicated for a small number of patients, posterior fixation and fusion is necessary for most. ${ }^{11}$ Frazier et al. demonstrated a clear association between preoperative spinal deformity and poor outcomes following decompression surgery, regardless of whether concurrent fusion was performed. ${ }^{6}$ Many authors have suggested that short or long fusion is necessary for DLS after decompression. On the other hand, in a study by Hosogane et al., in which patients with DLS were retrospectively divided into 2 surgical decompression groups depending on postopera-
Lumbar lordosis $\left.0^{\circ}\right)$

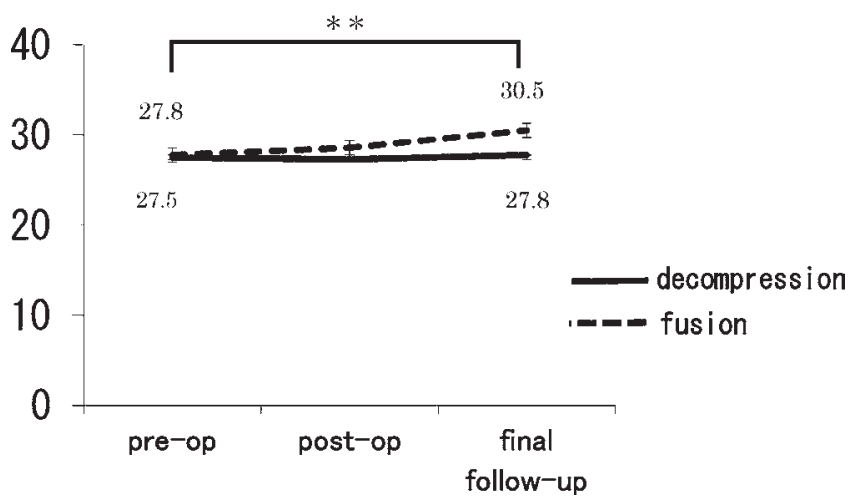

FIG. 5. Mean lumbar lordosis in the decompression and fusion groups (preoperatively, postoperatively, and at final follow-up). There is no significant change in lumbar lordosis in either group after surgery, nor is there a statistical difference in lordosis between the groups. Data are presented as the mean \pm SD. **not significant.

tive curve progression, there were no significant differences in revision rates between the groups regardless of curve progression, and none of the patients required arthrodesis due to curve progression. ${ }^{9}$

Chen et al. reported that decompression alone is appropriate for patients with moderate stenosis affecting up to 2 spinal levels and smaller curves, provided that there is no evidence of progression or sagittal imbalance, while decompression with limited fusion is appropriate when patients have moderate or severe stenosis affecting 3 or more levels, smaller curves, no progression of deformity, no imbalance, and no more than moderate risk factors for suboptimal outcomes. ${ }^{3}$ We hypothesized that further collapse, instability, and curve progression would not occur in patients in the decompression group, whose surgical treatment was determined based on our radiological parameters. In this study, the average lumbar curve progression in the decompression group was $1.2^{\circ}$. No patient in this group demonstrated Cobb angle progression greater than $10^{\circ}$, and the Cobb angle in the decompression group did not progress significantly after surgery.

In previous reports, reoperation rates after decompression surgery for spinal stenosis have varied from $5 \%$ to $23 \%$. Hansraj et al. reported that only $5 \%$ of patients required reoperation during a 5-year follow-up period, and Katz et al. reported that $23 \%$ of patients required revision surgery 7 to 10 years after decompression for lumbar canal stenosis. ${ }^{8}, 10$ In the decompression group, 2 patients underwent a second surgery, although only 1 patient (4\%) underwent a second surgery with instrumentation due to instability of the previously decompressed level. Most patients did not show further collapse or instability. The patients who underwent a lumbar spinous process-splitting technique versus open fenestration showed the same net change in JOA score and an equally maintained Cobb angle after surgery. Early-phase complications did not occur in the patients in this group. In the fusion group, 2 patients developed surgical site infections and underwent surgical debridement in the early phase. Two patients exhibited adjacent-segment degeneration and underwent additional 
surgeries. The complication rate in the decompression group was lower than that in the fusion group. The high infection rate and adjacent-segment degeneration in the fusion group might be due to the long operative time and use of instrumentation.

Transfeldt et al. compared the results of different surgical methods in patients who underwent decompression alone, decompression with limited fusion, and decompression with full curve fusion. ${ }^{17}$ They reported that the group receiving decompression alone had the lowest rate of complications and the best Oswestry Disability Index results, whereas the satisfaction was lowest in the decompressiononly group.

In this study, the JOA score improved in both groups, and the recovery rate based on the JOA score showed no difference between the groups. The patients assigned to the decompression group using our radiological parameters did well after surgery from the standpoint of radiological and clinical progression. The results of this study indicate that the evaluation of spinal instability by radiological parameters is appropriate for surgical decision-making.

One limitation is that the study does not evaluate whether the curve progressed, or whether the clinical outcomes and risk of complications of decompression surgery alone were appropriate for patients with positive radiological instability criteria and mild DLS. Furthermore, there is limited information on complications due to the small sample size, and further studies are needed to discuss whether fusion is required or decompression alone is appropriate for radiologically unstable segments.

\section{Conclusions}

This prospective study of patients with mild DLS treated by different surgical methods showed good results for both methods. The results of this study indicate that the radiological evaluation of spinal instability is appropriate for surgical decision-making.

\section{Acknowledgments}

We are grateful to Editage for the English language review.

\section{References}

1. Aota Y, Kumano K, Hirabayashi S: Postfusion instability at the adjacent segments after rigid pedicle screw fixation for degenerative lumbar spinal disorders. J Spinal Disord 8:464-473, 1995

2. Berven SH, Deviren V, Mitchell B, Wahba G, Hu SS, Bradford DS: Operative management of degenerative scoliosis: an evidence-based approach to surgical strategies based on clinical and radiographic outcomes. Neurosurg Clin N Am 18:261-272, 2007

3. Chen PG, Daubs MD, Berven S, Raaen LB, Anderson AT, Asch SM, et al: Surgery for degenerative lumbar scoliosis: the development of appropriateness criteria. Spine (Phila Pa 1976) 41:910-918, 2016

4. Daffner SD, Vaccaro AR: Adult degenerative lumbar scoliosis. Am J Orthop 32:77-82, 2003

5. Daubs MD, Lenke LG, Bridwell KH, Cheh G, Kim YJ, Stobbs G: Decompression alone versus decompression with limited fusion for treatment of degenerative lumbar scoliosis in the elderly patient. Evid Based Spine Care J 3:27-32, 2012

6. Frazier DD, Lipson SJ, Fossel AH, Katz JN: Association between spinal deformity and outcomes after decompression for spinal stenosis. Spine (Phila Pa 1976) 22:2025-2029, 1997

7. Gupta MC: Degenerative scoliosis. Options for surgical management. Orthop Clin North Am 34:269-279, 2003

8. Hansraj KK, Cammisa FP Jr, O'Leary PF, Crockett HC, Fras CI, Cohen MS, et al: Decompressive surgery for typical lumbar spinal stenosis. Clin Orthop Relat Res (384):10-17, 2001

9. Hosogane N, Watanabe K, Kono H, Saito M, Toyama Y, Matsumoto M: Curve progression after decompression surgery in patients with mild degenerative scoliosis. J Neurosurg Spine 18:321-326, 2013

10. Katz JN, Lipson SJ, Chang LC, Levine SA, Fossel AH, Liang MH: Seven- to 10-year outcome of decompressive surgery for degenerative lumbar spinal stenosis. Spine (Phila Pa 1976) 21:92-98, 1996

11. Liu W, Chen XS, Jia LS, Song DW: The clinical features and surgical treatment of degenerative lumbar scoliosis: a review of 112 patients. Orthop Surg 1:176-183, 2009

12. Marchesi DG, Aebi M: Pedicle fixation devices in the treatment of adult lumbar scoliosis. Spine (Phila Pa 1976) 17 (8 Suppl):S304-S309, 1992

13. Mochida J, Toh E, Nishimura K, Nomura T, Arima T: Percutaneous nucleotomy in lumbar disc herniation. Patient selection and role in various treatments. Spine (Phila Pa 1976) 18:2212-2217, 1993

14. Postacchini F: Surgical management of lumbar spinal stenosis. Spine (Phila Pa 1976) 24:1043-1047, 1999

15. Schwab F, Ungar B, Blondel B, Buchowski J, Coe J, Deinlein D, et al: Scoliosis Research Society-Schwab Adult Spinal Deformity Classification: a validation study. Spine (Phila Pa 1976) 37:1077-1082, 2012

16. Simmons ED: Surgical treatment of patients with lumbar spinal stenosis with associated scoliosis. Clin Orthop Relat Res (384):45-53, 2001

17. Transfeldt EE, Topp R, Mehbod AA, Winter RB: Surgical outcomes of decompression, decompression with limited fusion, and decompression with full curve fusion for degenerative scoliosis with radiculopathy. Spine (Phila Pa 1976) 35:1872-1875, 2010

18. Vaccaro AR, Ball ST: Indications for instrumentation in degenerative lumbar spinal disorders. Orthopedics 23:260273,2000

19. Watanabe K, Hosoya T, Shiraishi T, Matsumoto M, Chiba K, Toyama Y: Lumbar spinous process-splitting laminectomy for lumbar canal stenosis. Technical note. J Neurosurg Spine 3:405-408, 2005

\section{Disclosures}

The authors report no conflict of interest concerning the materials or methods used in this study or the findings specified in this paper.

\section{Author Contributions}

Conception and design: Masuda. Acquisition of data: Masuda. Analysis and interpretation of data: Masuda. Drafting the article: Masuda. Reviewed submitted version of manuscript: all authors.

\section{Correspondence}

Kenji Masuda: Yokohama City University School of Medicine, Yokohama, Japan.mashmash4952@msn.com. 